\title{
Comparison of Different Dynamic Contrast Enhanced-Magnetic Resonance Imaging Descriptors and Clinical Findings Among Breast Cancer Subtypes Determined Based on Molecular Assessment
}

\author{
Serap Dogan ${ }^{1,}$, , Soner Ozmen ${ }^{2}$, Bahadir $\mathrm{Oz}^{3}$, Hakan Imamoglu ${ }^{1}$, Guven Kahriman ${ }^{1}$, Gokmen \\ Zararsiz $^{4}$ and Mustafa Ozturk ${ }^{1}$ \\ ${ }^{1}$ Department of Radiology, Erciyes University Medical Faculty, Kayseri, Turkey \\ ${ }^{2}$ Department of Radiology, Gumushane State Hospital, Gumushane, Turkey \\ ${ }^{3}$ Department of General Surgery, Erciyes University Medical Faculty, Kayseri, Turkey \\ ${ }^{4}$ Department of Biostatistics, Erciyes University Medical Faculty, Kayseri, Turkey \\ "Corresponding author: Serap Dogan, Department of Radiology, Erciyes University Medical Faculty, 38090 Kayseri, Turkey. Tel: +90-3522076666-23781. Email: \\ drserapdogan@hotmail.com
}

Received 2017 December 08; Revised 2018 May 23; Accepted 2018 July 28.

\begin{abstract}
Background: Breast cancer is a heterogeneous disease with different molecular and histologic subtypes, clinical behaviors and prognosis. The same stage of disease and similar histopathological characteristics may show different treatment responses. Identification of breast cancer subtypes has become important for planning the targeted therapy and personalized management of patients.

Objectives: To compare the clinicopathologic findings, dynamic contrast enhanced-magnetic resonance imaging (DCE-MRI) characteristics and associated MRI findings among breast cancer molecular subtypes.

Patients and Methods: 267 pathologically proven invasive breast cancers in 263 patients were included. Clinicopathological findings, DCE-MRI findings and associated MRI findings were retrospectively evaluated and compared among breast cancer subtypes. Results: Invasive ductal carcinoma was the most common histological tumor type (87.6\%). There were 222 ( $83.1 \%$ ) masses and 45 (16.9\%) non-mass enhancements. The molecular subtypes were luminal A in 174 (65.1\%), luminal B in 45 (16.9\%), human epidermal growth factor receptor 2 (HER2) positive in 24 (9\%) and triple negative (TN) in 24 (9\%) of the lesions. Spiculated mass margin was significantly associated with luminal A breast cancer (45.2\%) and irregular shape was significantly more common in luminal A(86.3\%) and luminal B lesions (95.1\%) $(\mathrm{P}<0.001)$. Larger mass size $(\mathrm{P}=0.027)$, non-mass enhancement $(\mathrm{P}=0.005)$, perilesional + prepectoral edema and skin + perilesional + prepectoral edema were significantly associated with HER2 positive breast cancer $(\mathrm{P}=0.001)$. Higher histological grade, oval mass shape, circumscribed mass margin, intratumoral high/very high signal intensity on T2 weighted image (T2WI) were significantly associated with TN breast cancer $(\mathrm{P}<0.001)$.

Conclusion: Histological grade, size and morphological features of masses on DCE-MRI, intratumoral signal intensity on T2WI and edema pattern would be helpful to distinguish breast cancer subtypes.
\end{abstract}

Keywords: Breast Cancer, Dynamic-Contrast Enhanced Breast MRI, Molecular Subtypes

\section{Background}

Breast cancer is a heterogeneous disease with diverse clinical behaviors, histologic subtypes, treatment responses and outcome. Traditional criteria for treatment choices are the size of the tumor, histological grade, lymph node involvement, local invasion and distant metastases according to the American Joint Committee on Cancer's TNM staging classification (1). However, patients with the same stage of cancer and similar histopathological characteristics may show different clinical behavior and prog- nosis. Advances in gene expression analyses with DNA microarray technology have provided new molecular subtypes: Luminal A, luminal B, human epidermal growth factor receptor 2 (HER2)-enriched, and basal-like $(2,3)$. Immunohistochemical (IHC) staining is a reliable surrogate for these subtypes. Breast cancer subtypes are determined according to expression of the estrogen receptor (ER), the progesterone receptor (PR) and HER2 by IHC analysis. Clinically, the term "triple-negative (TN) cancer" is used as a substitude for basal-like breast cancer. Luminal A tumors have the best prognosis and tend to benefit from en- 
docrine therapy. Although luminal B subtypes have ERs, they are relatively insensitive to endocrine therapy and tend to be higher grade than luminal A tumors (4). HER2 positive tumors are likely to respond to targeted therapy (trastuzumab). TN breast cancers have agressive histologic characteristics and poor prognosis and do not respond to targeted or endocrine therapies (5). These differences in clinical behavior between molecular subtypes require personalized management of breast cancer patients.

Dynamic contrast enhanced magnetic resonance imaging (DCE-MRI) is an efficient imaging technique in evaluating breast cancer patients for preoperative surgery planning and treatment choices. The correlation of imaging findings with molecular subtypes of breast cancer is an emerging area of recent studies. There have been several reports on the morphologic features, diffusion weighted MRI characteristics and kinetic parameters of breast cancer molecular subtypes. Most previous studies were related to $\mathrm{TN}$ breast cancer because of the aggressive nature and unresponsiveness to the targeted therapies (69). There are few reports in the literature on the associated MR imaging features of different molecular subtypes of breast cancer $(10,11)$

\section{Objectives}

The aim of the this study was to compare clinicopathologic findings, DCE-MRI characteristics and associated MRI findings among breast cancer molecular subtypes.

\section{Patients and Methods}

\subsection{Patients}

This single institutional study was approved by the ethics committee of our university. Informed consent was waived due to retrospective design of the study. We retrospectively analyzed clinical records and breast MR images of 307 patients who were diagnosed with breast cancer at our instution. All MR images were obtained prior to treatment. Forty-four patients were excluded from the study. Of these, two had a nondiagnostic MRI, two had an axillary located lesion, six did not have a visible lesion on MRI, five had an unknown HER2 receptor status at histopathology, two were in lactating period, six had ductal carcinoma in situ, 16 received neoadjuvant therapy and five had a mass and non-mass enhancing lesion in the same breast. The remaining 263 patients were included in the present study. Four patients had contralateral breast cancer at the same time. Therefore, 267 invasive breast cancers were analyzed in this study.

\subsection{MR Imaging Technique}

MR imaging was performed on a $1.5 \mathrm{~T}$ scanner (Gyroscan Intera, Philips, Medical Systems, Best, the Netherlands) with breast surface coil. Imaging sequence parameters were T1 weighted turbo spin-echo (TSE) axial sequence (repetition time msec/ echotime msec, 550/11; slice thickness, $3 \mathrm{~mm}$; field of view [FOV], $34 \mathrm{~cm}$; matrix, $256 \times$ 204), T2 weighted TSE axial sequence (5000/120, slice thickness, $3 \mathrm{~mm}$, FOV, $34 \mathrm{~cm}$, matrix, $256 \times 180)$, short tau inversion recovery (STIR)sequence (5619/70, TI, $165 \mathrm{~ms}$, slice thickness, $3.3 \mathrm{~mm}$; FOV, $32 \mathrm{~cm}$; matrix, $256 \times 204)$. Threedimensional (3D) gradient-echo (GRE) THRIVE (T1 High Resolution Isotropic Volume Examination sequence) axial sequence with fat suppression (4.9/2.4, slice thickness, 1.1 $\mathrm{mm}$, interslice gap $0.3 \mathrm{~mm}$; FOV, $34 \mathrm{~cm}$, matrix, $348 \times 338$, flip angle, 10) was used for dynamic contrast-enhanced examination before and 6 times after intravenous administration of $0.1 \mathrm{mmol} / \mathrm{kg}$ of gadobutrol (Gadovist; Bayer Schering Pharma, Berlin, Germany). The contrast agent was injected into the antecubital vein with an injection rate of $2 \mathrm{~mL} / \mathrm{sec}$ and followed by an $20 \mathrm{~mL}$ saline flush. Dynamic contrast-enhanced image acquisition was started just after the injection. The acquisition time of each phase was 80 seconds. The total acquisition duration of MRI protocol was 20 minutes.

\subsection{Image Analysis}

MR images were retrospectively interpreted by two radiologists (SD, SO), experienced in breast MRI, blinded to the clinicopathologic data. Disagreements were solved by consensus. All enhancing lesions were evaluated by using the 5th edition of the American College of Radiology (ACR) breast imaging reporting and data system (BIRADS) MR lexicon (12). Mass lesions were interpreted in terms of size (the largest diameter), shape, margin, internal enhancement, multifocality (more than one lesion in the same quadrant with the index tumor), multicentricity (more than one lesion in the different quadrant from the index tumor), Non-mass lesions were evaluated for distribution and internal enhancement characteristics. All lesions were reviewed for time-signal intensity curve pattern (persistent, plateau, and washout) and intratumoral signal intensity on the T2 weighted image(T2WI) (classified as lower than-equivalent to, or higher than-very higher than that of the surrounding normal glandular tissue). Timesignal intensity curves were obtained by manually placing a region of interest (ROI), greater than 3 pixels, on the most intensely enhancing area of the lesion. During the ROI placement, special care was taken to avoid cystic-necrotic areas of the lesion or perilesional fatty tissue. ROI placement was repeated at least three times and the most suspicious curve was noted for each lesion. 
We furthermore analyzed all lesions for associated MR imaging findings such as skin or nipple invasion, chest wall or pectoralis muscle invasion, and edema. Skin or nipple invasion and chest wall or pectoralis muscle invasion were described as abnormal enhancement of these locations. Edema was subclassified as absent, perilesional edema, skin edema, perilesional + skin edema, perilesional + prepectoral edema, perilesional + prepectoral + skin edema.

\subsection{Histopathologic Assessment}

Pathological reports of breast-conserving surgery or mastectomy specimens were retrospectively analyzed to identify histological type, grade, and involvement of the lymph nodes. All pathologic specimens were obtained in the absence of systemic therapy. Histologic type was determined according to the World Health Organization classification system. Modified Bloom-Richardson grading system was used for histopathologic grading in patients with invasive ductal carcinoma- no otherwise specified (IDCNOS) (13). The descriptions of the lesions on MR images such as multifocality, multicenticity, skin or nipple invasion, chest wall or pectoralis muscle invasion were correlated with final histopathologic results. In patients with multifocal-multicentric disease or multiple regional nonmass enhancement, IHC staining findings of index lesion (the largest one) were analyzed. IHC staining was carried out with appropriate antibodies for ER (Novocastra, Newcastle upon Tyne, UK), PR(Novocastra) and HER2 (NeoMarkers, Fremont, CA, USA). ER and PR status were determined by using the Allred score (ranging from 0 to 8 ). The results were categorized as positive when the total score (the sum of the proportion score and immunointensity score) was 3 or more. In point of HER2 evaluation, membranous staining score 0 or $1+$ was considered as HER2-negative and score $3+$ was considered as HER2-positive. In tumors with a score $2+$, silver-enhanced in situ hybridization testing ( $\mathrm{SISH}$ ) was used to evaluate HER2/chromosome 17 ratio. A HER2/ chromosome 17 ratio more than 2.2 was considered positive.

Based on ER, PR and HER2 status, tumors were subclassified into the following four groups: luminal A (ER+ and/or PR+, HER2-), luminal B (ER+ and/or PR+, HER2+), HER2 positive (ER-, PR-, and HER2+), and TN (ER-, PR-, HER2-).

\subsection{Statistical Analysis}

Histogram and q-q values were analyzed and ShapiroWilk's test was applied to check the normality. Levene test was applied to test variance homogeneity. To compare the differences among groups, Chi-square analysis was used for categorical comparisons, one-way analysis of variance (ANOVA) or Kruskal-Wallis $\mathrm{H}$ test was used for continuous variables. Bonferroni adjusted $\mathrm{z}$ test and Tukey tests were applied for multiple comparisons. Logistic regression analyses were used to identify the risk factors of TN breast cancer. Odds ratios are calculated with 95\% confidence intervals. Significant variables at $\mathrm{P}<0.05$ were included into multiple model and backward elimination was applied using Wald statistic. Analyses were conducted using R 3.2.3 (www.r-project.org) software. A P value less than 0.05 was considered as statistically significant.

\section{Results}

\subsection{Clinicopathological Findings}

All patients were women and the patients' ages ranged from 21 to 86 years (mean, $50.07 \pm 11.82$ years). Clinicopathological characteristics of patients are summarized in Table 1. Invasive ductal carcinoma (IDC) was the most common histological tumor type (234/267, 87.6\%), followed by invasive lobular carcinoma (ILC) (17/267, 6.4\%). The other histological types were detected in a small number of patients such as musinous carcinoma in five, medullary carcinoma in three, papillary carcinoma in four, micropapillary carcinoma in one, cribriform carcinoma in one, secretory carcinoma in one, and neuroendocrine carcinoma in one patient. Therefore, statistical analysis could not be performed in terms of histological types.

The tumor subtypes by immunohistochemistry were luminal A in 174 (65.1\%), luminal B in 45 (16.9\%), HER2 positive in 24 (9\%), and TN in 24 (9\%) of the lesions. Regarding histological grade, grade 3 tumors were significantly higher in TN breast cancer $(81 \%)(\mathrm{P}<0.001)$ than that of luminal A (25\%), luminal B (31\%) lesions. Axillary lymph node involvement was seen most frequently in HER2 positive breast cancer $(15 / 24,62.5 \%)$. There were no significant differences in age $(\mathrm{P}=0.675)$ and axillary lymph node involvement among tumor subtypes $(\mathrm{P}=0.278)$.

\subsection{MR Imaging Findings}

Table 2 shows MR imaging characteristics of 267 invasive breast lesions. There were 222 (83.1\%) masses and 45 (16.9\%) non-mass enhancements. There was significant difference in lesion enhancement type (mass or non-mass) among subtypes $(\mathrm{P}=0.005)$. Mass enhancement was significantly associated with luminal A (146/174, 83.9\%) and luminal B $(41 / 45,91.1 \%)$ breast cancers compared with HER2 positive cancers (14/24, 58.3\%). Non-mass enhancement was significantly more common in HER2 positive breast cancers (10/24, 41.7\%) compared with luminal A (28/174, 16.1\%) and luminal B cancers $(4 / 45,8.9 \%)$ (Figure 1$)$. The median tumor size of mass lesions on MRI was significantly larger in 


\begin{tabular}{|c|c|c|c|c|c|}
\hline & Luminal A $(N=174)$ & Luminal $B(N=45)$ & HER2 positive $(N=24)$ & Triple-negative $(\mathrm{N}=\mathbf{2 4})$ & Pvalue \\
\hline Histological type & & & & & - \\
\hline IDC & $148(85.1)$ & $42(93.3)$ & $23(95.8)$ & $21(87.5)$ & \\
\hline ILC & $16(9.2)$ & $1(2.2)$ & $0(0)$ & $0(0)$ & \\
\hline Musinous Ca & $5(2.9)$ & $0(0)$ & $0(0)$ & $0(0)$ & \\
\hline Medullary Ca & $1(0.6)$ & $0(0)$ & $0(0)$ & $2(8.3)$ & \\
\hline Papillary Ca & $3(1.7)$ & $0(0)$ & $0(0)$ & $1(4.2)$ & \\
\hline Micropapillary Ca & $0(0)$ & $1(2.2)$ & $0(0)$ & $0(0)$ & \\
\hline Cribriform Ca & $0(0)$ & $0(0)$ & $1(4.2)$ & $0(0)$ & \\
\hline Secretory Ca & $1(0.6)$ & $0(0)$ & $0(0)$ & $0(0)$ & \\
\hline Neuroendocrine Ca & $0(0)$ & $1(2.2)$ & $0(0)$ & $0(0)$ & \\
\hline Age & $50.3 \pm 11.4$ & $48.1 \pm 11.8$ & $50.5 \pm 12.4$ & $51.2 \pm 14.1$ & 0.675 \\
\hline Grade (IDC-NOS) & & & & & $<0.001$ \\
\hline 1 & $32(21.6)$ & $4(9.5)$ & $0(0)$ & $0(0)$ & \\
\hline 2 & $79(53.4)$ & $25(59.5)$ & $11(47.8)$ & $4(19)$ & \\
\hline 3 & $37(25)$ & $13(31)$ & $12(52.2)$ & $17(81)$ & \\
\hline Axillary lymph node involvement & $99(56.9)$ & $26(57.8)$ & $15(62.5)$ & $9(37.5)$ & 0.278 \\
\hline
\end{tabular}

HER2 positive tumors compared with the luminal A cancers $(\mathrm{P}=0.027)$. Oval mass shape was significantly associated with TN breast cancer $(12 / 21,57.1 \%)$ compared with luminal A (17/146, 11.6\%) and luminal B (2/41, 4.9\%) cancers (P $<0.001)$. Irregular shape was more common in luminal $A$ (126/146, 86.3\%) and luminal B lesions (39/41, 95.1\%) than TN lesions $(8 / 21,38.1 \%)(\mathrm{P}<0.001)$ (Figure 2$)$. There was significant difference in mass margin among breast cancer subtypes $(\mathrm{P}<0.001)$. TN breast cancer more frequently had circumscribed margin $(11 / 21,52.4 \%)$ compared with luminal A (5/146, 3.4\%) and luminal B lesions (0/41, 0\%). Spiculated margin was associated with luminal A breast cancers (66/146, 45.2\%) compared with TN lesions (2/21, 9.5\%). HER2 positive breast cancer tended to be multifocal and multicentric $(5 / 14,35.7 \%$ and $3 / 14,21.4 \%)$ but this difference was not statistically significant $(\mathrm{P}=0.105)$ (Figure 3 ). The percentage of high/very high signal intensity on T2WI of TN breast cancers $(14 / 24,58.3 \%)$ was significantly higher than that of luminal A cancers $(35 / 174,20.1 \%)(P<0.001)$. Intratumoral signal intensity on T2WI $(\mathrm{P}=0.049)$ and mass margin $(\mathrm{P}<0.001)$ were independent predictors of the mass type TN breast cancer at multiple logistic regression analysis (Table 3 and Figure 4).

There was no statistically significant difference in internal enhancement pattern of mass $(\mathrm{P}=0.493)$ and nonmass lesion ( $\mathrm{P}=0.710)$, distribution of non-mass lesion ( $\mathrm{P}$
$=0.474)$ and time-signal intensity curve pattern $(\mathrm{P}=0.134)$ among breast cancer subtypes.

When all mass and non-mass lesions were evaluated together regarding associated MR imaging findings, there was no statistically significant difference involving skin or nipple invasion ( $\mathrm{P}=0.934)$ and chest wall or pectoralis muscle invasion $(\mathrm{P}=0.148)$ among breast cancers subtypes (Table 4). Perilesional + prepectoral edema and skin + perilesional + prepectoral edema were significantly associated with HER2 positive breast cancer $(4 / 24,16.7 \%$ and $6 / 24,25 \%$ ) compared with luminal A cancers (4/174, 2.3\% and $10 / 174,5.7 \%)(P=0.001)$. However, when mass and nonmass lesions were analyzed separately, chest wall or pectoralis muscle invasion was significantly associated with TN breast cancer $(1 / 3,33.3 \%)$ compared with luminal A cancers $(0 / 28,0 \%)(P=0.003)$ in non-mass lesions. In addition, perilesional + prepectoral edema was significantly associated with HER2 positive tumors $(3 / 14,21.4 \%)$ compared with luminal A cancers $(4 / 146,2.7 \%)(\mathrm{P}=0.049)$ in mass lesions.

\section{Discussion}

Identification of breast cancer subtypes has become important for planning the targeted-endocrine therapy and optimal management of patients. The present study 


\begin{tabular}{|c|c|c|c|c|c|}
\hline Characteristics & Luminal A $(N=174)$ & Luminal $B(N=45)$ & HER2 positive $(N=24)$ & Triple-negative $(\mathrm{N}=\mathbf{2 4})$ & Pvalue \\
\hline Lesion type & & & & & 0.005 \\
\hline Mass & $146(83.9)$ & $41(91.1)$ & $14(58.3)$ & $21(87.5)$ & \\
\hline Non-mass & $28(16.1)$ & $4(8.9)$ & $10(41.7)$ & $3(12.5)$ & \\
\hline Size & $23(18-30)$ & $27(18-35)$ & $33.5(26-38)$ & $26(23-40)$ & 0.027 \\
\hline Shape & & & & & $<0.001$ \\
\hline Round & $3(2.1)$ & $0(0)$ & $0(0)$ & $1(4.8)$ & \\
\hline Oval & $17(11.6)$ & $2(4.9)$ & $3(21.4)$ & $12(57.1)$ & \\
\hline Irregular & $126(86.3)$ & $39(95.1)$ & $11(78.6)$ & $8(38.1)$ & \\
\hline Margin & & & & & $<0.001$ \\
\hline Circumscribed & $5(3.4)$ & $0(0)$ & $2(14.3)$ & $11(52.4)$ & \\
\hline Irregular & $75(51.4)$ & $28(68.3)$ & $8(57.1)$ & $8(38.1)$ & \\
\hline Spiculated & $66(45.2)$ & $13(31.7)$ & $4(28.6)$ & $2(9.5)$ & \\
\hline Internal enhancement & & & & & 0.493 \\
\hline Homogeneous & $8(5.5)$ & $3(7.3)$ & $0(0)$ & $0(0)$ & \\
\hline Heterogeneous & $104(71.2)$ & $31(75.6)$ & $11(78.6)$ & $13(61.9)$ & \\
\hline Rim enhancement & $34(23.3)$ & $7(17.1)$ & $3(21.4)$ & $8(38.1)$ & \\
\hline Additional lesion & & & & & 0.105 \\
\hline Unifocal & $101(69.2)$ & $28(68.3)$ & $6(42.9)$ & $18(85.7)$ & \\
\hline Multifocal & $17(11.6)$ & $5(12.2)$ & $5(35.7)$ & $2(9.5)$ & \\
\hline \multicolumn{6}{|c|}{ Non-Mass } \\
\hline Distribution & & & & & 0.474 \\
\hline Focal & $2(7.1)$ & $1(25)$ & $1(10)$ & $1(33.3)$ & \\
\hline Segmental & $11(39.3)$ & $2(50)$ & $0(0)$ & $1(33.3)$ & \\
\hline Regional & $4(14.3)$ & $0(0)$ & $3(30)$ & $0(0)$ & \\
\hline Multiple regional & $4(14.3)$ & $0(0)$ & $3(30)$ & $0(0)$ & \\
\hline Diffuse & $7(25)$ & $1(25)$ & $3(30)$ & $1(33.3)$ & \\
\hline Internal enhancement & & & & & 0.710 \\
\hline Homogeneous & $3(10.7)$ & $0(0)$ & $1(10)$ & $1(33.3)$ & \\
\hline Heterogeneous & $22(78.6)$ & $3(75)$ & $6(60)$ & $1(33.3)$ & \\
\hline Clumped & $2(7.1)$ & $1(25)$ & $2(20)$ & $1(33.3)$ & \\
\hline Clustered ring & $1(3.6)$ & $0(0)$ & $1(10)$ & $0(0)$ & \\
\hline Time-intensity curves & & & & & 0.134 \\
\hline Persistent & $14(8)$ & $2(4.4)$ & $0(0)$ & $1(4.2)$ & \\
\hline Plateau & $107(61.5)$ & $20(44.4)$ & $17(70.8)$ & $14(58.3)$ & \\
\hline Washout & $53(30.5)$ & $23(51.1)$ & $7(29.2)$ & $9(37.5)$ & \\
\hline Intratumoral SI on T2WI & & & & & $<0.001$ \\
\hline Low/equal & $139(79.9)$ & $33(73.3)$ & $14(58.3)$ & $10(41.7)$ & \\
\hline High/very high & $35(20.1)$ & $12(26.7)$ & $10(41.7)$ & $14(58.3)$ & \\
\hline
\end{tabular}

Abbreviation: T2WI, T2 weighted image.

${ }^{\mathrm{a}}$ Time-intensity curves and intratumoral SI on T2WI were evaluated for all mass and non-mass lesions.

${ }^{b}$ Values are expressed as No. (\%) or median (25th - 75th percentiles). 


\begin{tabular}{|c|c|c|c|c|}
\hline \multirow[t]{3}{*}{ Variable } & \multicolumn{4}{|c|}{ Triple-negative breast cancer/ non- triple-negative breast cancer } \\
\hline & \multicolumn{2}{|c|}{ Univariate } & \multicolumn{2}{|c|}{ Multivariate } \\
\hline & OR $(95 \% \mathrm{CI})$ & Pvalue & OR $(95 \% \mathrm{CI})$ & Pvalue \\
\hline Size & $1.02(0.99-1.05)$ & 0.093 & - & - \\
\hline Shape & & & - & - \\
\hline Round & 1.0 & & & \\
\hline Oval & $1.63(0.15-17.50)$ & 0.684 & & \\
\hline Irregular & $0.13(0.01-1.46)$ & 0.100 & & \\
\hline \multicolumn{5}{|l|}{ Margin } \\
\hline Circumscribed & 1.0 & & 1.0 & \\
\hline Irregular & $0.04(0.01-0.15)$ & $<0.001$ & $0.05(0.015-0.174)$ & $<0.001$ \\
\hline Spiculated & $0.01(0.003-0.08)$ & $<0.001$ & $0.02(0.004-0.124)$ & $<0.001$ \\
\hline Internal enhancement & & & - & \\
\hline Rim enhancement & 1.0 & & & \\
\hline Heterogeneous & $0.49(0.19-1.25)$ & 0.138 & & \\
\hline Additional lesion & & & - & \\
\hline Unifocal & 1.0 & & & \\
\hline Multifocal & $0.55(0.12-2.53)$ & 0.448 & & \\
\hline Multicentric & $0.19(0.02-1.48)$ & 0.114 & & \\
\hline Time-intensity curves & & & - & \\
\hline Persistent & 1.0 & & & \\
\hline Plateau & $1.31(0.15-10.90)$ & 0.802 & & \\
\hline Washout & $1.50(0.17-13.09)$ & 0.710 & & \\
\hline \multicolumn{5}{|l|}{ Intratumoral SI on T2WI } \\
\hline Low/equal & 1.0 & & 1.0 & \\
\hline High/very high & $4.62(1.83-11.66)$ & 0.001 & $2.78(1.01-8.30)$ & 0.049 \\
\hline Skin or nipple invasion & $1.38(0.16-11.84)$ & 0.766 & - & - \\
\hline Chest wall or pectoralis muscle invasion & $1.96(0.21-17.61)$ & 0.548 & - & - \\
\hline
\end{tabular}

showed that there were significant differences in histological grade, lesion type (mass-nonmass), size, shape, margin features of mass lesions, intratumoral signal intensity on T2WI, and edema pattern among breast cancer molecular subtypes.

Our findings revealed that the most common histological type was IDC. The other histological types were diagnosed in a small number of patients. Uematsu et al. (8) found that metaplastic and medullary carcinoma were significantly associated with TN breast cancer. Costantini et al. (6) reported that there were no statistically differences among the molecular subtypes in terms of histological type. Special type breast cancers such as cribriform, metaplastic, medullary carcinoma are relatively rare histo- logical subtypes. We think that further studies with a great number of patients are required to clarify the relationship between special histological type and molecular subtypes of breast cancer.

Luminal $\mathrm{A}$ is the most common molecular subtype and typically has the best prognosis (14). Luminal B subtype is more aggressive than luminal A cancers (15). Our study revealed that the majority of luminal A and luminal $\mathrm{B}$ tumors were seen as irregular shaped mass with irregular/spiculated margin and heterogeneous enhancement. There was no significant difference between imaging findings of luminal A and luminal B breast cancers. To the best of our knowledge, there is only one published report distinguishing the MRI appearances of luminal A and luminal 


\begin{tabular}{|c|c|c|c|c|c|}
\hline & Luminal A & Luminal B & HER2-positive & Triple-negative & Pvalue \\
\hline Total (mass and non-mass) & 174 & 45 & 24 & 24 & \\
\hline Skin or nipple invasion & $10(5.7)$ & $3(6.7)$ & $2(8.3)$ & $2(8.3)$ & 0.934 \\
\hline Chest wall or pectoralis muscle invasion & $2(1.1)$ & $2(4.4)$ & $1(4.2)$ & $2(8.3)$ & 0.148 \\
\hline Edema & & & & & 0.001 \\
\hline Absent & $41(23.6)$ & $9(20)$ & $2(8.3)$ & $2(8.3)$ & \\
\hline Perilesional & $82(47.1)$ & $24(53.3)$ & $7(29.2)$ & $14(58.3)$ & \\
\hline Skin & $7(4)$ & $0(0)$ & $0(0)$ & $0(0)$ & \\
\hline Perilesional + skin & $30(17.2)$ & $7(15.6)$ & $5(20.8)$ & $1(4.2)$ & \\
\hline Perilesional + prepectoral & $4(2.3)$ & $2(4.4)$ & $4(16.7)$ & $3(12.5)$ & \\
\hline Perilesional + prepectoral + skin & $10(5.7)$ & $3(6.7)$ & $6(25)$ & $4(16.7)$ & \\
\hline Mass & 146 & 41 & 14 & 21 & \\
\hline Skin or nipple invasion & $4(2.7)$ & $2(4.9)$ & $1(7.1)$ & $1(4.8)$ & 0.779 \\
\hline Chest wall or pectoralis muscle invasion & $2(1.4)$ & $2(4.9)$ & $1(7.1)$ & $1(4.8)$ & 0.375 \\
\hline Edema & & & & & 0.049 \\
\hline Absent & $38(26)$ & $8(19.5)$ & $2(14.3)$ & $2(9.5)$ & \\
\hline Perilesional & $69(47.3)$ & $23(56.1)$ & $5(35.7)$ & $14(66.7)$ & \\
\hline Skin & $5(3.4)$ & $0(0)$ & $0(0)$ & $0(0)$ & \\
\hline Perilesional + skin & $24(16.4)$ & $5(12.2)$ & $3(21.4)$ & $0(0)$ & \\
\hline Perilesional + prepectoral & $4(2.7)$ & $2(4.9)$ & $3(21.4)$ & $3(14.3)$ & \\
\hline Perilesional + prepectoral + skin & $6(4.1)$ & $3(7.3)$ & $1(7.1)$ & $2(9.5)$ & \\
\hline Non-Mass & 28 & 4 & 10 & 3 & \\
\hline Skin or nipple invasion & $6(21.4)$ & $1(25)$ & $1(10)$ & $1(33.3)$ & 0.788 \\
\hline Chest wall or pectoralis muscle invasion & $0(0)$ & $0(0)$ & $0(0)$ & $1(33.3)$ & 0.003 \\
\hline Edema & & & & & 0.246 \\
\hline Absent & $3(10.7)$ & $1(25)$ & $0(0)$ & $0(0)$ & \\
\hline Perilesional & $13(46.4)$ & $1(25)$ & $2(20)$ & $0(0)$ & \\
\hline Skin & $2(7.1)$ & $0(0)$ & $0(0)$ & $0(0)$ & \\
\hline Perilesional + skin & $6(21.4)$ & $2(50)$ & $2(20)$ & $1(33.3)$ & \\
\hline Perilesional + prepectoral & $0(0)$ & $0(0)$ & $1(10)$ & $0(0)$ & \\
\hline Perilesional + prepectoral + skin & $4(14.3)$ & $0(0)$ & $5(50)$ & $2(66.6)$ & \\
\hline
\end{tabular}

Abbreviation: HER2, human epidermal growth factor receptor 2.

${ }^{\mathrm{a}}$ Values are expressed as No. (\%).

B tumors. Kato et al. (16) reported that rim enhancement was significantly more common in luminal B cancers than luminal A cancers.

A spiculated mass margin was reported to be associated with lower histologic grade and would be a major feature for differentiating between ER-positive and ERnegative cancers $(16,17)$. Spiculated mass margin was associated with luminal A breast cancers compared with TN lesions in our study. Irregular shaped, irregular/spicular marginated, heterogeneous enhanced mass with lower histological grade may suggest ER + breast cancer.

HER2 positive breast cancers tend to have poor prognosis (18). HER2 positive breast cancers constitute 10\% - 15\% of the all breast cancers (19). HER2 positive subtype was seen in $9 \%$ of tumors in the present study. The present study revealed that a larger tumor size and non-mass enhancement were significantly more common in HER2 positive tumors. Also, multifocality and multicentricity were more common in patients with HER2 positive breast cancer than the other subtypes. To our knowledge, there are a 

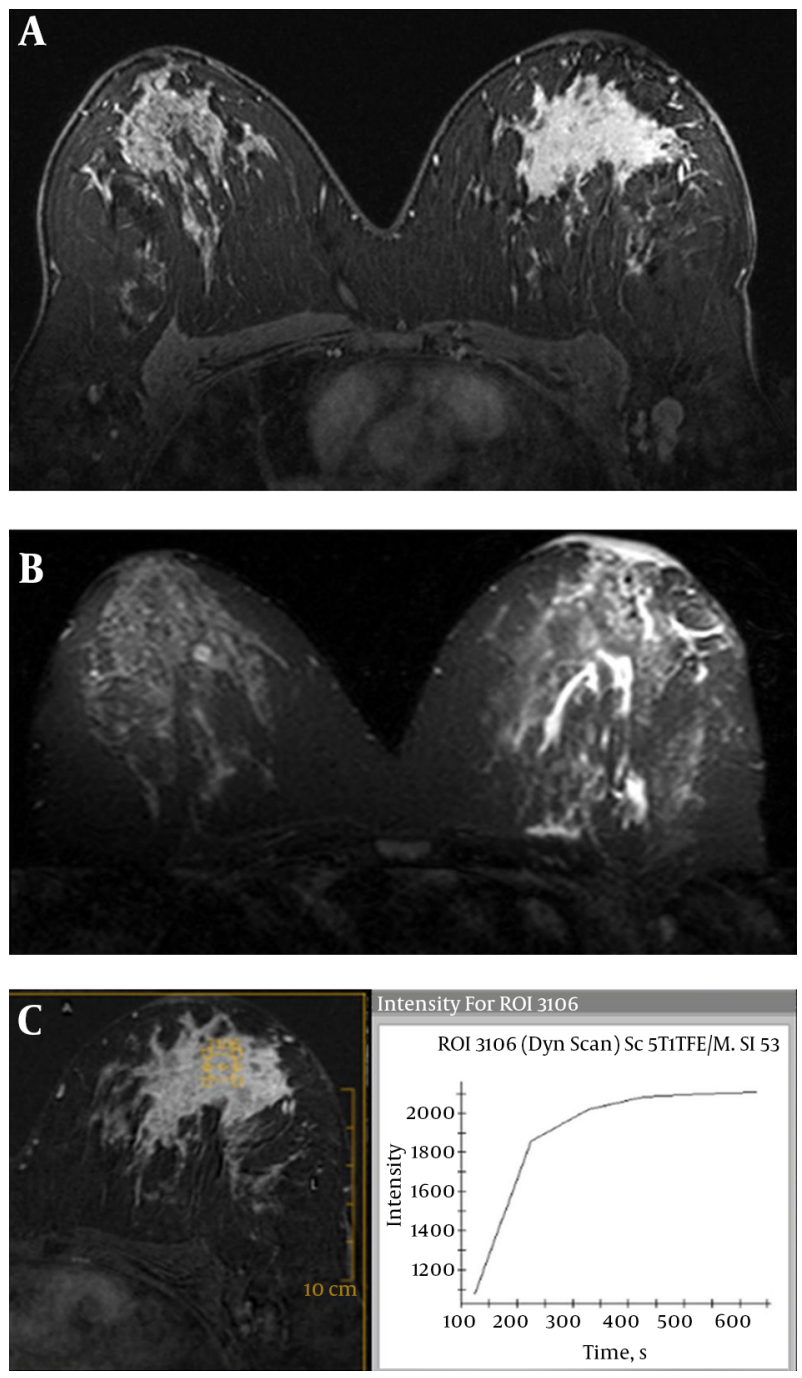

Figure 1. Human epidermal growth factor receptor 2 (HER2) positive breast cancer (invasive ductal carcinoma) of the left breast in a 38-year-old woman. A, Axial contrast-enhanced three-dimensional gradient-echo T1 high resolution isotropic volume examination (THRIVE) image with fat suppression shows diffuse non-mass lesion with heterogeneous internal enhancement. B, Axial short tau inversion recovery (STIR) image reveals skin + perilesional + prepectoral edema. C, Corresponding time-intensity curve indicates plateau kinetic.

limited number of reports on the specific appearances of HER2 positive tumors. Grimm et al. (10) reported that multicentric or multifocal disease was significantly more frequent in luminal B and HER2 positive subtypes.

Edema pattern was classified into five subgroups and we found that perilesional + prepectoral edema and skin + perilesional + prepectoral edema were associated with HER2 positive breast cancer compared with the luminal A cancers. Alili et al. (20) reported that perilesional edema was more common in HER positive cancers than
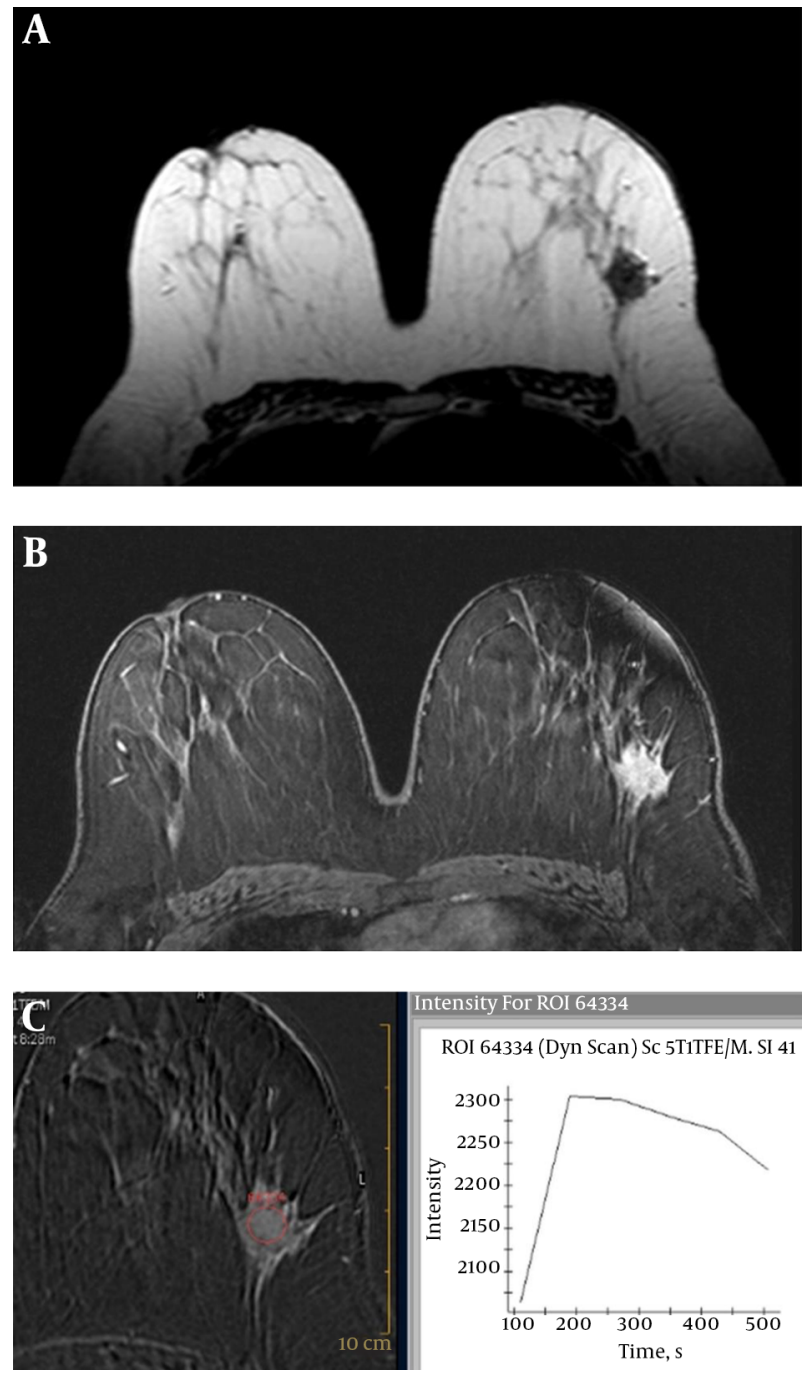

Figure 2. Luminal A breast cancer (invasive ductal carcinoma) of the left breast in a 52-year-old woman. A, Axial T2 weighted turbo spin-echo image shows a low intensity mass with irregular shape. B, Axial contrast-enhanced three-dimensional gradient-echo T1 high resolution isotropic volume examination (THRIVE) image with fat suppression (second post-contrast phase image of dynamic series) shows a $32 \mathrm{~mm}$ mass with spiculated margin and heterogeneous enhancement. C, Corresponding time-intensity curve indicates washout kinetic.

luminal subtypes. Our results support their study. The present study is the first study to evaluate edema pattern as five subgroups and we described significant difference in edema pattern.

TN breast cancers have the worst prognosis of all tumor types and they are responsible for a large percentage of deaths, due to aggressive characteristics and absence of specific treatment $(7,21)$. In our study, TN breast cancers were associated with a higher histological grade, oval mass shape, circumscribed mass margin and high/very high sig- 

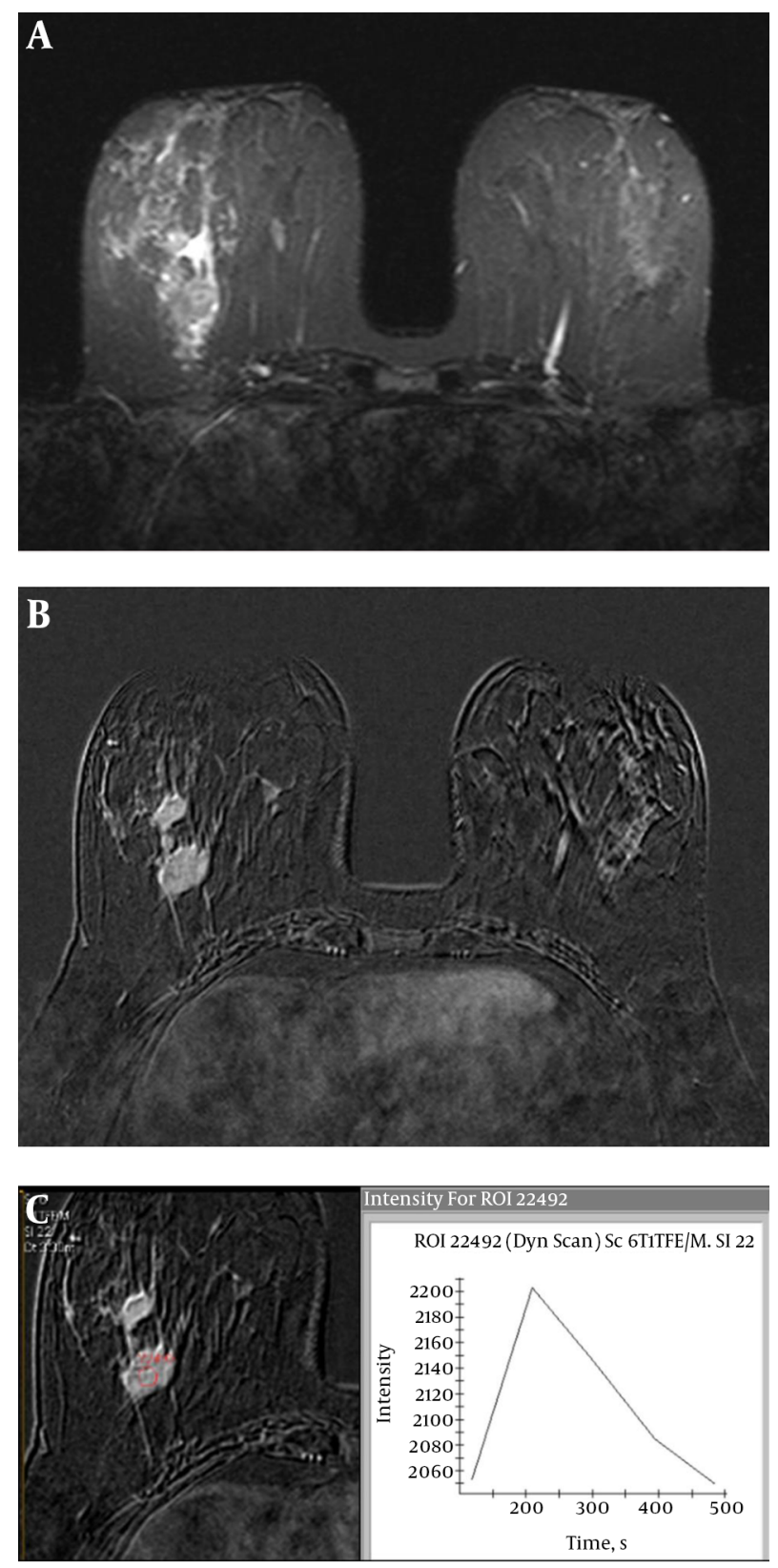

Figure 3. Human epidermal growth factor receptor 2 (HER2) positive breast cancer (invasive ductal carcinoma) of the right breast in a 48-year-old woman. A, Axial short tau inversion recovery (STIR) image shows perilesional edema around the mass lesions. B, Axial second post-contrast phase substracted image of dynamic examination shows multifocal heterogeneous enhanced mass lesions. C, Time-intensity curve indicates washout kinetic.

nal intensity on T2WI. Multiple logistic regression analysis identified the intratumoral signal intensity on T2WI and mass margin as independent predictors of the $\mathrm{TN}$ breast cancer. High intratumoral signal intensity on T2WI, corresponding to necrosis is associated with a poor prognos-
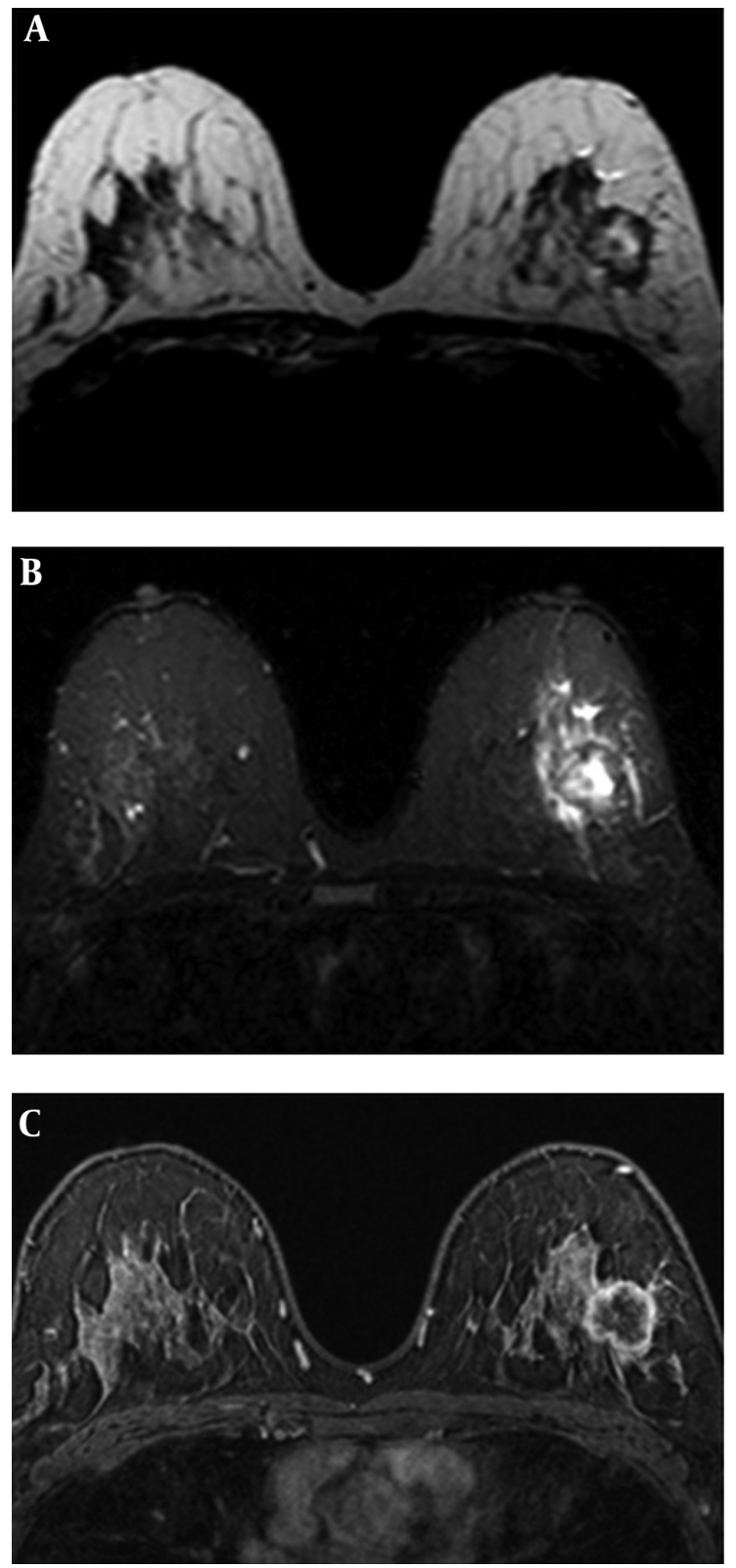

Figure 4. Triple-negative breast cancer (invasive ductal carcinoma) of the left breast in a 42-year-old woman. A, Axial T2 weighted turbo spin-echo image shows a mass with intratumoral high/very high intensity. B, Axial short tau inversion recovery (STIR) image reveals perilesional edema. C, Axial contrast-enhanced three-dimensional gradient-echo T1 high resolution isotropic volume examination (THRIVE) image with fat suppression (second post-contrast phase image of dynamic series) shows a $33 \mathrm{~mm}$ oval mass with a circumscribed margin and rim enhancement.

tic factor in invasive breast cancers (22). Higher histological grade with oval, circumscribed mass and high intratumoral signal intensity on T2WI may suggest TN tumor. Although oval shape and circumscribed margins are usually 
suggestive of benign breast lesions, rim enhancement may alert the radiologist to the presence of an aggressive lesion $(23,24)$. Rim enhancement was reported as a significantly associated finding with TN tumors (8). In the present study, rim enhancement was seen most frequently in TN subtype.

Uematsu et al. (8) reported that $66 \%$ of TN tumors were unifocal, with the remainder being multifocal; there was no multicentricity. Dogan et al. (9) later reported that multicentric disease was seen in $23 \%$ of the TN breast cancers. In our study, most of the TN cancers were unifocal (85.7\%), but multifocal and multicentric disease were seen in $9.5 \%$ and $4.8 \%$ of the TN cancers, respectively. There are diverse results about the age of TN breast cancer patients. Costantini et al. (6) found that a younger age was significantly associated with the TN subtype. In the present study, there was no significant difference between age and breast cancer subtypes.

The present study revealed that there were no significant differences in distribution and internal enhancement pattern of the non-mass lesion, and time-signal intensity curve pattern among breast cancer subtypes. Non-mass enhancement is a relatively rare presentation of breast cancers. Distribution and internal enhancement features of non-mass lesions were previously reported by Youk et al. (7) $(8.4 \%, 23 / 271)$, Uematsu et al. (8) (23.8\%, 42/176), and Kato et al. (16) $(14.3 \%, 14 / 98)$. We reported imaging features of 45 (16.9\%) non-mass enhancing lesions and there were no significant differences in distribution and internal enhancement features of non-mass lesions in agreement with previous reports $(7,16)$. We also found that chest wall or pectoralis muscle invasion was significantly associated with TN breast cancer compared with luminal A cancers in nonmass lesions. However, patient numbers were too small in these groups. Further studies with a large number of patients are needed to analyze the features of non-mass lesions in different breast cancer subtypes. Time-signal intensity curves have been used to differentiate benign and malignant lesions. Different curve patterns were reported in each molecular subtypes previously (6-9) but there is no conclusive data about the possibility of reliably differentiating breast cancer subtypes based on the time-signal intensity curves.

The major limitation of the present study was the retrospective study design. Another limitation was that the numbers of HER2 positive and TN tumors were small.

In conclusion, histological grade, size and morphological features of masses on DCE-MRI, intratumoral signal intensity on T2WI and edema pattern would be useful to differentiate breast cancer molecular subtypes. The combined analysis of histopathologic findings and DCE-MRI findings may provide prediction of molecular subtypes of breast cancer to plan the personalized therapy and opti- mal management of patients. Further prospective studies with a large number of subjects are needed to evaluate diagnostic performances of MR imaging for differentiating tumor subtypes.

\section{Footnotes}

Authors' Contributions: Study concept and design, Serap Dogan, Soner Ozmen; acquisition of data, Serap Dogan, Soner Ozmen; analysis and interpretation of data, Serap Dogan, Soner Ozmen; drafting of the manuscript, Serap Dogan, Hakan Imamoglu; critical revision of the manuscript for important intellectual content, Guven Kahriman; statistical analysis, Gokmen Zararsiz; administrative, technical, and material support, Hakan Imamoglu; study supervision, Mustafa Ozturk.

Financial Disclosure: The authors declare that they have no conflict of interest.

Funding/Support: This study was not funded by any funding source.

\section{References}

1. Edge SB, Byrd DR, Compton CC, Fritz AG, Greene FL, Trotti A.AJCC cancer staging manual. 7th ed. New York, NY: Springer; 2009. p. 347-76.

2. Perou CM, Sorlie T, Eisen MB, van de Rijn M, Jeffrey SS, Rees CA, et al. Molecular portraits of human breast tumours. Nature. 2000;406(6797):747-52. doi: 10.1038/35021093. [PubMed: 10963602].

3. Huber KE, Carey LA, Wazer DE. Breast cancer molecular subtypes in patients with locally advanced disease: impact on prognosis, patterns of recurrence, and response to therapy. Semin Radiat Oncol. 2009;19(4):204-10. doi: 10.1016/j.semradonc.2009.05.004. [PubMed: 19732684].

4. Mazurowski MA, Zhang J, Grimm LJ, Yoon SC, Silber JI. Radiogenomic analysis of breast cancer: Luminal B molecular subtype is associated with enhancement dynamics at MR imaging. Radiology. 2014;273(2):365-72. doi: 10.1148/radiol.14132641. [PubMed: 25028781].

5. Dent R, Trudeau M, Pritchard KI, Hanna WM, Kahn HK, Sawka CA, et al. Triple-negative breast cancer: Clinical features and patterns of recurrence. Clin Cancer Res. 2007;13(15 Pt 1):4429-34. doi: 10.1158/10780432.CCR-06-3045. [PubMed: 17671126].

6. Costantini M, Belli P, Distefano D, Bufi E, Matteo MD, Rinaldi P, et al. Magnetic resonance imaging features in triple-negative breast cancer: Comparison with luminal and HER2-overexpressing tumors. Clin Breast Cancer. 2012;12(5):331-9. doi: 10.1016/j.clbc.2012.07.002. [PubMed: 23040001].

7. Youk JH, Son EJ, Chung J, Kim JA, Kim EK. Triple-negative invasive breast cancer on dynamic contrast-enhanced and diffusion-weighted MR imaging: Comparison with other breast cancer subtypes. Eur Radiol. 2012;22(8):1724-34. doi: 10.1007/s00330-012-2425-2. [PubMed: 22527371].

8. Uematsu T, Kasami M, Yuen S. Triple-negative breast cancer: Correlation between MR imaging and pathologic findings. Radiology. 2009;250(3):638-47. doi: 10.1148/radiol.2503081054. [PubMed: 19244039].

9. Dogan BE, Gonzalez-Angulo AM, Gilcrease M, Dryden MJ, Yang WT. Multimodality imaging of triple receptor-negative tumors with mammography, ultrasound, and MRI. AJR Am J Roentgenol. 2010;194(4):1160-6. doi: 10.2214/AJR.09.2355. [PubMed: 20308526]. 
10. Grimm LJ, Johnson KS, Marcom PK, Baker JA, Soo MS. Can breast cancer molecular subtype help to select patients for preoperative MR imaging? Radiology. 2015;274(2):352-8. doi: 10.1148/radiol.14140594. [PubMed: 25325325].

11. Ha R, Jin B, Mango V, Friedlander L, Miloshev V, Malak S, et al. Breast cancer molecular subtype as a predictor of the utility of preoperative MRI. AJR Am J Roentgenol. 2015;204(6):1354-60. doi: 10.2214/AJR.14.13666. [PubMed: 26001248].

12. American College of Radiology . Breast imaging and reporting and data system (ACR BI-RADS®Atlas). 5th ed. Reston, VA: American College of Radiology; 2013.

13. Rakha EA, El-Sayed ME, Lee AH, Elston CW, Grainge MJ, Hodi Z, et al. Prognostic significance of Nottingham histologic grade in invasive breast carcinoma. J Clin Oncol. 2008;26(19):3153-8. doi: 10.1200/JCO.2007.15.5986. [PubMed: 18490649].

14. Lam SW, Jimenez CR, Boven E. Breast cancer classification by proteomic technologies: Current state of knowledge. Cancer Treat Rev 2014;40(1):129-38. doi: 10.1016/j.ctrv.2013.06.006. [PubMed: 23891266].

15. Kyndi M, Sorensen FB, Knudsen H, Overgaard M, Nielsen HM, Over gaard J, et al. Estrogen receptor, progesterone receptor, HER-2, and response to postmastectomy radiotherapy in high-risk breast cancer: The Danish Breast Cancer Cooperative Group. J Clin Oncol. 2008;26(9):1419-26. doi: 10.1200/JCO.2007.14.5565. [PubMed: $18285604]$.

16. Kato F, Kudo K, Yamashita H, Wang J, Hosoda M, Hatanaka KC, et al. Differences in morphological features and minimum apparent diffusion coefficient values among breast cancer subtypes using 3-tesla MRI. Eur J Radiol. 2016;85(1):96-102. doi: 10.1016/j.ejrad.2015.10.018. [PubMed: 26724653].

17. Lee SH, Cho N, Kim SJ, Cha JH, Cho KS, Ko ES, et al. Correlation between high resolution dynamic MR features and prognostic factors in breast cancer. Korean J Radiol. 2008;9(1):10-8. doi: 10.3348/kjr.2008.9.1.10.
[PubMed: 18253071]. [PubMed Central: PMC2627175].

18. Carey LA, Dees EC, Sawyer L, Gatti L, Moore DT, Collichio F, et al. The triple negative paradox: primary tumor chemosensitivity of breast cancer subtypes. Clin Cancer Res. 2007;13(8):2329-34. doi: 10.1158/10780432.CCR-06-1109. [PubMed: 17438091].

19. Sorlie T, Tibshirani R, Parker J, Hastie T, Marron JS, Nobel A, et al. Repeated observation of breast tumor subtypes in independent gene expression data sets. Proc Natl Acad Sci U S A. 2003;100(14):8418-23. doi: 10.1073/pnas.0932692100. [PubMed: 12829800]. [PubMed Central: PMC166244].

20. Alili C, Pages E, Curros Doyon F, Perrochia H, Millet I, Taourel P. Correlation between MR imaging - prognosis factors and molecular classification of breast cancers. Diagn Interv Imaging. 2014;95(2):235-42. doi: 10.1016/j.diii.2014.01.002. [PubMed: 24525088].

21. Boudin L, Chabannon C, Sfumato P, Sabatier R, Bertucci F, Tarpin C, et al. Immunohistochemical subtypes predict survival in metastatic breast cancer receiving high-dose chemotherapy with autologous haematopoietic stem cell transplantation. EurJCancer. 2016;57:118-26. doi: 10.1016/j.ejca.2016.01.005. [PubMed: 26918737].

22. Jimenez RE, Wallis T, Visscher DW. Centrally necrotizing carcinomas of the breast: A distinct histologic subtype with aggressive clinical behavior. Am J Surg Pathol. 2001;25(3):331-7. doi: 10.1097/00000478200103000-00007. [PubMed: 11224603].

23. Trop I, LeBlanc SM, David J, Lalonde L, Tran-Thanh D, Labelle M, et al. Molecular classification of infiltrating breast cancer: Toward personalized therapy. Radiographics. 2014;34(5):1178-95. doi: 10.1148/rg.345130049. [PubMed: 25208275].

24. Schnall MD, Blume J, Bluemke DA, DeAngelis GA, DeBruhl N, Harms $S$, et al. Diagnostic architectural and dynamic features at breast MR imaging: Multicenter study. Radiology. 2006;238(1):42-53. doi: 10.1148/radiol.2381042117. [PubMed: 16373758]. 